\title{
ON THE EDGEWORTH EXPANSION FOR ELEMENTARY POLYNOMIALS BASED ON TRIMMED SAMPLES
}

\author{
YURI V. BorovSKIKH AND N. C. WEBER
}

\begin{abstract}
This paper develops a one term Edgeworth expansion under minimal conditions for elementary symmetric polynomials of any degree based on trimmed samples. These statistics are special cases of trimmed $U$-statistics and natural extensions of the trimmed mean.
\end{abstract}

\section{INTRODUCTION AND RESUltS}

Let $X_{1}, \ldots, X_{n}$ be independent and identically distributed, real-valued, random variables with distribution function $F$ and let $X_{n 1} \leqslant \cdots \leqslant X_{n n}$ denote the order statistics of the $X_{i}^{\prime} s$. We shall consider the behaviour of trimmed versions of the elementary symmetric polynomials studied in $[\mathbf{1 2}, \mathbf{8}, \mathbf{9}, \mathbf{2 1}]$, among others.

Consider the trimmed U-statistic sum of the form

$$
U(\alpha, \beta)=\left(\begin{array}{c}
k_{\alpha \beta} \\
m
\end{array}\right)^{-1} \sum_{k_{\alpha}+1 \leqslant i_{1}<\cdots<i_{m} \leqslant k_{\beta}} h\left(X_{n i_{1}}, \ldots, X_{n i_{m}}\right)
$$

with the kernel

$$
h\left(x_{1}, \ldots, x_{m}\right)=x_{1} \cdots x_{m}, m \geqslant 1,
$$

where $0 \leqslant \alpha<\beta \leqslant 1$ are any fixed numbers, $k_{\alpha \beta}=k_{\beta}-k_{\alpha}, k_{\alpha}=[\alpha n], k_{\beta}=[\beta n]$ and $[\cdot]$ denotes the integer part.

If $\alpha=0$ and $\beta=1$ then $U(0,1)$ corresponds to the ordinary elementary symmetric polynomial of degree $m$ which is a $U$-statistic with product kernel based on the full sample. Limit theorems, Berry-Esseen bounds, Edgeworth espansions and large deviation results have been established for this class of statistic. See, for example, $[8,10,4,6]$.

For $m=1,(1)$ gives the $(\alpha, \beta)$ trimmed sample mean

$$
\bar{X}(\alpha, \beta)=\left(k_{\beta}-k_{\alpha}\right)^{-1} \sum_{i=k_{\alpha}+1}^{k_{\beta}} X_{n i} .
$$

The asymptotic normality of the trimmed mean was established in [3] and many properties were established in the theory of robust estimation. The approximation problems

Received 4th October, 2004

Copyright Clearance Centre, Inc. Serial-fee code: 0004-9727/05 \$A2.00+0.00. 
connected with sharpening the rate of convergence to the normal distribution of $\bar{X}(\alpha, \beta)$ have been explored in many papers including $[11,13,14,15,19,22,23,24,25]$.

For the general class of $U$-statistics, the asymptotic normality of the trimmed statistic was established in [17]. For fixed $m \geqslant 1$ we shall obtain conditions under which the 1-term Edgeworth expansion holds for the trimmed elementary symmetric polynomials given in (1).

We need the following notation. Write $F^{-1}(u)=\inf \{x: F(x) \geqslant u\}, 0<u \leqslant 1$, for the left-continuous inverse-function of $F$ and $F_{n}(x)$ for the empirical distribution function. The $\gamma$ th quantile of $F$ is $\xi_{\gamma}=F^{-1}(\gamma)$. The sample estimate of $\xi_{\gamma}$ is $\bar{\xi}_{\gamma}=F_{n}^{-1}(\gamma)=X_{n k_{\gamma}}$.

Let

$$
\mu=(\beta-\alpha)^{-1} \int_{\alpha}^{\beta} F^{-1}(u) d u
$$

and $w_{\gamma}=n \gamma-[n \gamma]$ with $\gamma=\alpha, \beta$. Let $W_{i}, i=1, \ldots, n$, denote $X_{i}$ winsorised outside of $\left(\xi_{\alpha}, \xi_{\beta}\right]$, that is

$$
W_{i}=\xi_{\alpha} I\left(X_{i} \leqslant \xi_{\alpha}\right)+X_{i} I\left(\xi_{\alpha}<X_{i} \leqslant \xi_{\beta}\right)+\xi_{\beta} I\left(X_{i}>\xi_{\beta}\right),
$$

where $I(A)$ is the indicator of the event $A$. Then $W_{i} \stackrel{d}{=} Q\left(U_{i}\right), i=1, \ldots, n$, where $U_{i}$ are independent random variables uniformly distributed on $(0,1)$ and

$$
Q(u)=\xi_{\alpha} I(u \leqslant \alpha)+F^{-1}(u) I(\alpha<u \leqslant \beta)+\xi_{\beta} I(u>\beta) .
$$

Furthermore, let $W_{n i}, i=1, \ldots, n$ denote the order statistics corresponding to $W_{1}, \ldots, W_{n}$. Then

$$
W_{n i}=\xi_{\alpha} I\left(i \leqslant N_{\alpha}\right)+X_{n i} I\left(N_{\alpha}<i \leqslant N_{\beta}\right)+\xi_{\beta} I\left(i>N_{\beta}\right),
$$

where $N_{\gamma}=\sum_{i=1}^{n} I\left(X_{i} \leqslant \xi_{\gamma}\right)$ with $\gamma=\alpha, \beta$. Note that

$$
\begin{aligned}
& N_{\gamma} \stackrel{d}{=} \sum_{i=1}^{n} I\left(U_{i} \leqslant \gamma\right) . \text { Let } \\
& \begin{aligned}
& v=\int_{0}^{1} Q(u) d u, \quad \sigma^{2}=\int_{0}^{1}(Q(u)-v)^{2} d u \\
& \gamma_{2}=-\alpha^{2} \frac{1}{f\left(\xi_{\alpha}\right)}\left(v-\xi_{\alpha}\right)^{2}+(1-\beta)^{2} \frac{1}{f\left(\xi_{\beta}\right)}\left(v-\xi_{\beta}\right)^{2}+\frac{m-1}{\beta-\alpha} \frac{\sigma^{4}}{\mu}, \\
& \gamma_{3}=\int_{0}^{1}(Q(u)-v)^{3} d u, \quad \lambda_{1}=\gamma_{3} / \sigma^{3}, \quad \lambda_{2}=\gamma_{2} / \sigma^{3}, \\
& \lambda_{3}=6 \sigma^{-1}\left\{\left(\xi_{\alpha}-\mu\right) w_{\alpha}-\left(\xi_{\beta}-\mu\right) w_{\beta}-\frac{\alpha(1-\alpha)}{2 f\left(\xi_{\alpha}\right)}+\frac{\beta(1-\beta)}{2 f\left(\xi_{\beta}\right)}\right. \\
&\left.\quad+\frac{m-1}{2(\beta-\alpha) \mu}\left[\alpha\left(\xi_{\alpha}-\mu\right)^{2}+(1-\beta)\left(\xi_{\beta}-\mu\right)^{2}-(v-\mu)^{2}\right]\right\} .
\end{aligned}
\end{aligned}
$$


For $x \in R$ let

$$
\begin{gathered}
F_{\sigma}(x)=P\left(\frac{\sqrt{n}(\beta-\alpha)}{m \mu^{m-1} \sigma}\left(U(\alpha, \beta)-\mu^{m}\right) \leqslant x\right), \\
G(x)=\Phi(x)-\frac{\phi(x)}{6 \sqrt{n}}\left(\lambda_{1}+3 \lambda_{2}\left(x^{2}-1\right)+\lambda_{3}\right),
\end{gathered}
$$

where $\Phi$ is the standard normal distribution function, $\phi=\Phi^{\prime}$.

THEOREM 1. Assume that $f=F^{\prime}$ exists in neighbourhoods of $\xi_{\alpha}$ and $\xi_{\beta}$ where it satisfies a Lipschitz condition. Further assume that $f\left(\xi_{\alpha}\right)>0$ and $f\left(\xi_{\beta}\right)>0$. Then

$$
\sup _{x}\left|F_{\sigma}(x)-G(x)\right|=O\left((\ln n)^{5 / 4} n^{-3 / 4}\right)
$$

as $n \rightarrow \infty$.

To studentise $U(\alpha, \beta)$ we need an estimate of $\sigma^{2}$. We shall use

$$
S_{n}^{2}=\frac{k_{\alpha}}{n} X_{n k_{\alpha}}^{2}+\frac{1}{n} \sum_{i=k_{\alpha}+1}^{k_{\beta}-1} X_{n i}^{2}+\frac{n-k_{\beta}+1}{n} X_{n k_{\beta}}^{2}-v_{n}^{2}
$$

where

$$
v_{n}=\frac{k_{\alpha}}{n} X_{n k_{\alpha}}+\frac{1}{n} \sum_{i=k_{\alpha}+1}^{k_{\beta}-1} X_{n i}+\frac{n-k_{\beta}+1}{n} X_{n k_{\beta}} .
$$

For $x \in R$ let

$$
\begin{aligned}
& F_{s}(x)=P\left(\frac{\sqrt{n}(\beta-\alpha)}{m \mu^{m-1} S_{n}}\left(U(\alpha, \beta)-\mu^{m}\right) \leqslant x\right), \\
& H(x)=\Phi(x)+\frac{\phi(x)}{6 \sqrt{n}}\left(\left(2 x^{2}+1\right) \lambda_{1}+3\left(x^{2}+1\right) \lambda_{2}-\lambda_{3}\right) .
\end{aligned}
$$

THEOREM 2. Assume that the conditions of Theorem 1 are satisfied. Then

$$
\sup _{x}\left|F_{s}(x)-H(x)\right|=O\left((\ln n)^{5 / 4} n^{-3 / 4}\right)
$$

as $n \rightarrow \infty$.

\section{ProOFS}

We begin with following lemma which gives a useful representation of $U(\alpha, \beta)$.

\section{LEMMA 1.}

$$
U(\alpha, \beta)-\mu^{m}=\sum_{r=1}^{m} \frac{m(m-1) \cdots(m-r+1)}{k_{\alpha \beta}\left(k_{\alpha \beta}-1\right) \cdots\left(k_{\alpha \beta}-r+1\right)} \cdot \mu^{m-r} \cdot S_{r}(\alpha, \beta),
$$

where

$$
S_{r}(\alpha, \beta)=\sum(-1)^{r+i_{1}+\cdots+i_{r}} \prod_{\ell=1}^{r}\left(\ell^{i_{\ell}} i_{\ell} !\right)^{-1} \prod_{\ell=1}^{r} \pi_{\ell}^{i_{\ell}},
$$


where the summation is over all non-negative integers $i_{1}, \ldots, i_{r}$ satisfying $\sum_{\ell=1}^{r} \ell i_{\ell}=r$, in addition,

$$
\pi_{\ell}=\sum_{p=0}^{\ell}\left(\begin{array}{l}
\ell \\
p
\end{array}\right)(-\mu)^{\ell-p} \sigma_{p}(\alpha, \beta)
$$

where

$$
\sigma_{p}(\alpha, \beta)=\sum_{i=1}^{n} W_{i}^{p}-k_{\alpha} \xi_{\alpha}^{p}-\left(n-k_{\beta}\right) \xi_{\beta}^{p}+J_{p}(\alpha)-\bar{J}_{p}(\alpha)-J_{p}(\beta)+\bar{J}_{p}(\beta)
$$

with, for $\gamma=\alpha, \beta$,

$$
J_{p}(\gamma)=I\left(k_{\gamma}<N_{\gamma}\right) \sum_{i=k_{\gamma}+1}^{N_{\gamma}}\left(X_{n i}^{p}-\xi_{\gamma}^{p}\right), \bar{J}_{p}(\gamma)=I\left(k_{\gamma}>N_{\gamma}\right) \sum_{i=N_{\gamma}+1}^{k_{\gamma}}\left(X_{n i}^{p}-\xi_{\gamma}^{p}\right) .
$$

PROOF: First we have Hoeffding's decomposition

$$
U(\alpha, \beta)-\mu^{m}=\sum_{r=1}^{m} \frac{m(m-1) \cdots(m-r+1)}{k_{\alpha \beta}\left(k_{\alpha \beta}-1\right) \cdots\left(k_{\alpha \beta}-r+1\right)} \mu^{m-r} S_{r}(\alpha, \beta),
$$

where

$$
S_{r}(\alpha, \beta)=\sum_{\boldsymbol{k}_{\alpha}+1 \leqslant i_{1}<\cdots<i_{r} \leqslant k_{\beta}}\left(X_{n i_{1}}-\mu\right) \cdots\left(X_{n i_{r}}-\mu\right) .
$$

Further, by Waring's formula, (see for example, [7]), we obtain for $S_{r}(\alpha, \beta)$ the representation (9) with

$$
\pi_{\ell}=\sum_{i=k_{\alpha}+1}^{k_{\beta}}\left(X_{n i}-\mu\right)^{\ell}
$$

Hence, it is necessary to prove that this $\pi_{\ell}$ has the form (10). Indeed,

$$
\pi_{\ell}=\sum_{i=k_{\alpha}+1}^{k_{\beta}} \sum_{p=0}^{\ell}\left(\begin{array}{l}
\ell \\
p
\end{array}\right)(-\mu)^{\ell-p} X_{n i}^{p}=\sum_{p=0}^{\ell}\left(\begin{array}{l}
\ell \\
p
\end{array}\right)(-\mu)^{\ell-p} \sigma_{p}(\alpha, \beta)
$$

where

$$
\sigma_{p}(\alpha, \beta)=\sum_{i=k_{\alpha}+1}^{k_{\beta}} X_{n i}^{p}
$$

Using (4) and (6) we can write

$$
\sum_{i=1}^{n} W_{i}^{p}=\sum_{i=1}^{n} W_{n i}^{p}=N_{\alpha} \xi_{\alpha}^{p}+\sum_{i=N_{\alpha}+1}^{N_{\beta}} X_{n i}^{p}+\left(n-N_{\beta}\right) \xi_{\beta}^{p}
$$


that is,

$$
\sum_{i=N_{\alpha}+1}^{N_{\beta}} X_{n i}^{p}=\sum_{i=1}^{n} W_{i}^{p}-N_{\alpha} \xi_{\alpha}^{p}-\left(n-N_{\beta}\right) \xi_{\beta}^{p} .
$$

Furthermore, we have

$$
\begin{aligned}
\sum_{i=k_{\alpha}+1}^{k_{\beta}} X_{n i}^{p}=\sum_{i=N_{\alpha}+1}^{N_{\beta}} X_{n i}^{p}+J_{p}(\alpha)-\bar{J}_{p}(\alpha)+\bar{J}_{p}(\beta) & -J_{p}(\beta) \\
& +\left(N_{\alpha}-k_{\alpha}\right) \xi_{\alpha}^{p}+\left(k_{\beta}-N_{\beta}\right) \xi_{\beta}^{p}
\end{aligned}
$$

From (13)-(15) we obtain (11) and hence (10). Lemma 1 is proved.

Let $U_{1}=1 /(\sigma \sqrt{n}) \sum_{i=1}^{n} g\left(X_{i}\right)$, where

$$
g\left(X_{i}\right)=W_{i}-v, U_{2}=1 /(\sigma n \sqrt{n}) \sum_{1 \leqslant i<j \leqslant n} h\left(X_{i}, X_{j}\right)
$$

and

$$
\begin{aligned}
h\left(X_{i}, X_{j}\right)= & -\left(I\left(X_{i} \leqslant \xi_{\alpha}\right)-\alpha\right)\left(I\left(X_{j} \leqslant \xi_{\alpha}\right)-\alpha\right) \frac{1}{f\left(\xi_{\alpha}\right)} \\
& +\left(I\left(X_{i} \leqslant \xi_{\beta}\right)-\beta\right)\left(I\left(X_{j} \leqslant \xi_{\beta}\right)-\beta\right) \frac{1}{f\left(\xi_{\beta}\right)}+\frac{(m-1)}{(\beta-\alpha) \mu}\left(W_{i}-v\right)\left(W_{j}-v\right) .
\end{aligned}
$$

LEMma 2. Suppose that the conditions of Theorem 1 are satisfied. Then

$$
\begin{aligned}
& \frac{\sqrt{n}(\beta-\alpha)}{m \mu^{m-1} \sigma}\left(U(\alpha, \beta)-\mu^{m}\right)=U_{1}+U_{2}+\frac{\lambda_{3}}{6 \sqrt{n}} \\
& \quad+\frac{1}{n \sqrt{n}}\left|\sum_{i=1}^{n}\left(I\left(X_{i} \leqslant \xi_{\alpha}\right)-\alpha\right)\right|^{3 / 2} R_{n}+\frac{1}{n \sqrt{n}}\left|\sum_{i=1}^{n}\left(I\left(X_{i} \leqslant \xi_{\beta}\right)-\beta\right)\right|^{3 / 2} R_{n}+\bar{R}_{n},
\end{aligned}
$$

where $R_{n}$ and $\bar{R}_{n}$ satisfy

$$
P\left(\left|R_{n}\right|>c \sqrt{\ln n}\right)=O\left(n^{-d}\right), \quad P\left(\left|\bar{R}_{n}\right|>c(\ln n)^{3 / 2} n^{-1}\right)=O\left(n^{-d}\right)
$$

as $n \rightarrow \infty$ for some sufficiently large, positive constants $c$ and $d$ not depending on $n$.

Proof: We shall follow the approach in [13] and [11] to obtain sharp approximations for $J_{p}(\gamma)$ and $\bar{J}_{p}(\gamma)$ in (12) by functions of $N_{\gamma}$ for $0<\gamma<1$ and any integer $p \geqslant 1$. Let $U_{n 1} \leqslant \cdots \leqslant U_{n n}$ be the order statistics corresponding to the independent random variables $U_{1}, \ldots, U_{n}$ uniformly distributed on $(0,1)$.

Estimating $J_{p}(\gamma)$. Under the conditions of the theorem

$$
\begin{aligned}
J_{p}(\gamma) & \stackrel{d}{=} I\left(k_{\gamma}<N_{\gamma}\right) \sum_{i=k_{\gamma}+1}^{N_{\gamma}}\left[\left(F^{-1}\left(U_{n i}\right)\right)^{p}-\left(F^{-1}(\gamma)\right)^{p}\right] \\
& =I\left(k_{\gamma}<N_{\gamma}\right)\left\{\frac{p \xi_{\gamma}^{p-1}}{f\left(\xi_{\gamma}\right)} \sum_{i=k_{\gamma}+1}^{N_{\gamma}}\left(U_{n i}-\gamma\right)+r(\gamma)\right\},
\end{aligned}
$$


where

$$
|r(\gamma)| \leqslant c \sum_{i=k_{\gamma}+1}^{N_{\gamma}}\left(U_{n i}-\gamma\right)^{2}
$$

and the constant $c$ can depend on $p, \gamma$ and $F$. Conditional on $N_{\gamma}$ the order statistics $U_{n i}, 1 \leqslant i \leqslant N_{\gamma}$, are distributed as the order statistics from a sample of size $N_{\gamma}$ from the uniform distribution on $(0, \gamma)$. Therefore for $i=1, \ldots, N_{\gamma}$

$$
\mu_{i}(\gamma)=E\left(U_{n i} \mid N_{\gamma}\right)=\frac{\gamma i}{N_{\gamma}+1}, \quad \sigma_{i}^{2}(\gamma)=E\left(\left(U_{n i}-\mu_{i}(\gamma)\right)^{2} \mid N_{\gamma}\right)=\frac{\gamma^{2} i\left(N_{\gamma}-i+1\right)}{\left(N_{\gamma}+1\right)^{2}\left(N_{\gamma}+2\right)}
$$

and in (16)

$$
\begin{aligned}
& \sum_{i=k_{\gamma}+1}^{N_{\gamma}}\left(U_{n i}-\gamma\right)=-\frac{\gamma}{2\left(N_{\gamma}+1\right)}\left(N_{\gamma}-k_{\gamma}\right)\left(N_{\gamma}-k_{\gamma}+1\right)+\sum_{i=k_{\gamma}+1}^{N_{\gamma}}\left(U_{n i}-\mu_{i}(\gamma)\right) \\
& \sum_{i=k_{\gamma}+1}^{N_{\gamma}}\left(U_{n i}-\gamma\right)^{2} \leqslant 2 \gamma^{2} \frac{\left(N_{\gamma}-k_{\gamma}\right)^{3}}{\left(N_{\gamma}+1\right)^{2}}+2 \sum_{i=k_{\gamma}+1}^{N_{\gamma}}\left(U_{n i}-\mu_{i}(\gamma)\right)^{2}
\end{aligned}
$$

Denote for $i=k_{\gamma}+1, \ldots, N_{\gamma}$

$$
\eta_{i}=\left(U_{n i}-\mu_{i}(\gamma)\right) / \sigma_{i}(\gamma)
$$

and note that

$$
\sigma_{i}^{2}(\gamma) \leqslant \gamma^{2} \frac{N_{\gamma}-k_{\gamma}}{\left(N_{\gamma}+1\right)^{2}}
$$

For $\eta_{i}$ we can write (see, for example, Lemma 3.1.1 in [20]),

$$
P\left(\left|\eta_{i}\right|>c \sqrt{\ln n} \mid N_{\gamma}\right)=O\left(n^{-d}\right)
$$

uniformly for $k_{\gamma}+1 \leqslant i \leqslant N_{\gamma}$ with some positive constants $c$ and $d$ which do not depend on $n$. Furthermore

$$
\begin{aligned}
\left|\sum_{i=k_{\gamma}+1}^{N_{\gamma}}\left(U_{n i}-\mu_{i}(\gamma)\right)\right| & \leqslant\left(N_{\gamma}-k_{\gamma}\right) \max _{k_{\gamma}+1 \leqslant i \leqslant N_{\gamma}}\left|U_{n i}-\mu_{i}(\gamma)\right| \\
& \leqslant \gamma \frac{1}{\left(N_{\gamma}+1\right)}\left(N_{\gamma}-k_{\gamma}\right)^{3 / 2} \max _{k_{\gamma}+1 \leqslant i \leqslant N_{\gamma}}\left|\eta_{i}\right|
\end{aligned}
$$

and

$$
\begin{aligned}
\sum_{i=k_{\gamma}+1}^{N_{\gamma}}\left(U_{n i}-\mu_{i}(\gamma)\right)^{2} & \leqslant\left(N_{\gamma}-k_{\gamma}\right) \max _{k_{\gamma}+1 \leqslant i \leqslant N_{\gamma}}\left(U_{n i}-\mu_{i}(\gamma)\right)^{2} \\
& \leqslant \gamma^{2} \frac{1}{\left(N_{\gamma}+1\right)^{2}}\left(N_{\gamma}-k_{\gamma}\right)^{2} \max _{k_{\gamma}+1 \leqslant i \leqslant N_{\gamma}} \eta_{i}^{2}
\end{aligned}
$$


Combining (16)-(20) we find

$$
J_{p}(\gamma)=-I\left(k_{\gamma}<N_{\gamma}\right) \frac{\left(N_{\gamma}-k_{\gamma}\right)^{2}}{n} \frac{p}{2} \frac{\xi_{\gamma}^{p-1}}{f\left(\xi_{\gamma}\right)}+\frac{1}{n}\left|N_{\gamma}-k_{\gamma}\right|^{3 / 2} r_{n}+\frac{1}{n^{2}}\left(N_{\gamma}-k_{\gamma}\right)^{2} r_{n}^{2}
$$

with $P\left(\left|r_{n}\right|>c \sqrt{\ln n}\right)=O\left(n^{-d}\right)$.

Estimating $\bar{J}_{p}(\gamma)$. By analogy with (16) we write

$$
\bar{J}_{p}(\gamma) \stackrel{d}{=} I\left(k_{\gamma}>N_{\gamma}\right)\left\{\frac{p \xi_{\gamma}^{p-1}}{f\left(\xi_{\gamma}\right)} \sum_{i=N_{\gamma}+1}^{k_{\gamma}}\left(U_{n i}-\gamma\right)+\bar{r}(\gamma)\right\},
$$

where

$$
|\bar{r}(\gamma)| \leqslant c \sum_{i=N_{\gamma}+1}^{k_{\gamma}}\left(U_{n i}-\gamma\right)^{2} .
$$

Note that now conditional on $N_{\gamma}$ the order statistics $U_{n i}, N_{\gamma}+1 \leqslant i \leqslant n$ are distributed as the order statistics of a sample of size $n-N_{\gamma}$ from a uniform distribution on $(\gamma, 1)$. Hence for $i=N_{\gamma}+1, \ldots, n$

$$
\begin{aligned}
& \bar{\mu}_{i}(\gamma)=E\left(U_{n i} \mid N_{\gamma}\right)=\gamma+(1-\gamma) \frac{\left(i-N_{\gamma}\right)}{n-N_{\gamma}+1}, \\
& \bar{\sigma}_{i}^{2}(\gamma)=E\left(\left(U_{n i}-\bar{\mu}_{i}(\gamma)\right)^{2} \mid N_{\gamma}\right)=\frac{(1-\gamma)^{2}\left(i-N_{\gamma}\right)(n-i+1)}{\left(n-N_{\gamma}+1\right)^{2}\left(n-N_{\gamma}+2\right)}
\end{aligned}
$$

and if $N_{\gamma}+1 \leqslant i \leqslant k_{\gamma}$ then

$$
\bar{\sigma}_{i}^{2}(\gamma) \leqslant(1-\gamma)^{2} \frac{k_{\gamma}-N_{\gamma}}{\left(n-N_{\gamma}+1\right)^{2}}
$$

and

$$
P\left(\left|\bar{\eta}_{i}\right|>c \sqrt{\ln n} \mid N_{\gamma}\right)=O\left(n^{-d}\right),
$$

where $\bar{\eta}_{i}=\left(U_{n i}-\bar{\mu}_{i}(\gamma)\right) / \bar{\sigma}_{i}(\gamma)$. Further, by analogy with (17)-(21) we obtain from (22)

$$
\bar{J}_{p}(\gamma)=I\left(k_{\gamma}>N_{\gamma}\right) \frac{\left(N_{\gamma}-k_{\gamma}\right)^{2}}{n} \frac{p}{2} \frac{\xi_{\gamma}^{p-1}}{f\left(\xi_{\gamma}\right)}+\frac{1}{n}\left|N_{\gamma}-k_{\gamma}\right|^{3 / 2} \bar{r}_{n}+\frac{1}{n^{2}}\left(N_{\gamma}-k_{\gamma}\right)^{2} \bar{r}_{n}^{2}
$$

with $P\left(\left|r_{n}\right|>c \sqrt{\ln n}\right)=O\left(n^{-d}\right)$.

Combining (10), (11), (21) and (23) we find

$$
\begin{aligned}
\pi_{\ell}=\sum_{i=1}^{n}\left(W_{i}-\mu\right)^{\ell} & -k_{\alpha}\left(\xi_{\alpha}-\mu\right)^{\ell}-\left(n-k_{\beta}\right)\left(\xi_{\beta}-\mu\right)^{\ell} \\
& -\frac{\left(N_{\alpha}-k_{\alpha}\right)^{2}}{n} \frac{\ell\left(\xi_{\alpha}-\mu\right)^{\ell-1}}{2 f\left(\xi_{\alpha}\right)}+\frac{\left(N_{\beta}-k_{\beta}\right)^{2}}{n} \frac{\ell\left(\xi_{\beta}-\mu\right)^{\ell-1}}{2 f\left(\xi_{\beta}\right)} \\
& +\left(\frac{1}{n}\left|N_{\alpha}-k_{\alpha}\right|^{3 / 2}+\frac{1}{n}\left|N_{\beta}-k_{\beta}\right|^{3 / 2}\right) r_{n \ell} \\
& +\left(\frac{1}{n^{2}}\left(N_{\alpha}-k_{\alpha}\right)^{2}+\frac{1}{n^{2}}\left(N_{\beta}-k_{\beta}\right)^{2}\right) r_{n \ell}^{2}
\end{aligned}
$$


where $r_{n \ell}$ satisfies $P\left(\max _{1 \leqslant \ell \leqslant m}\left|r_{n \ell}\right|>c \sqrt{\ln n}\right\}=O\left(n^{-d}\right)$. Note that in (24) by Bernstein's inequality

$$
P\left(\left|N_{\gamma}-k_{\gamma}\right|>c \sqrt{n \ln n}\right)=O\left(n^{-d}\right) .
$$

Furthermore, from (8)

$$
U(\alpha, \beta)-\mu^{m}=\frac{m}{k_{\alpha \beta}} \mu^{m-1} \pi_{1}+\frac{m(m-1)}{k_{\alpha \beta}\left(k_{\alpha \beta}-1\right)} \mu^{m-2} \frac{1}{2}\left(\pi_{1}^{2}-\pi_{2}\right)+T_{n}(\alpha, \beta),
$$

where

$$
T_{n}(\alpha, \beta)=\sum_{r=3}^{m} \frac{m(m-1) \cdots(m-r+1)}{k_{\alpha \beta}\left(k_{\alpha \beta}-1\right) \cdots\left(k_{\alpha \beta}-r+1\right)} \mu^{m-r} S_{r}(\alpha, \beta) .
$$

EsTimating $T_{n}(\alpha, \beta)$. We shall show that

$$
P\left(\left|\sqrt{n} T_{n}(\alpha, \beta)\right|>c(\ln n)^{3 / 2} n^{-1}\right)=O\left(n^{-d}\right) .
$$

According to $(9) S_{r}(\alpha, \beta)$ is a polynomial of degree $r$ on $r$ variables $\pi_{1}, \ldots, \pi_{r}$. Each of these variables we can estimate, with the help of the representation (24). At first let $\ell=1$. Since $E W_{1}=v=\alpha \xi_{\alpha}+(\beta-\alpha) \mu+(1-\beta) \xi_{\beta}$, then in (24)

$$
\begin{aligned}
\pi_{1}=\sum_{i=1}^{n}\left(W_{i}-v\right)+\left(\xi_{\alpha}-\mu\right) w_{\alpha} & -\left(\xi_{\beta}-\mu\right) w_{\beta} \\
& -\frac{\left(N_{\alpha}-k_{\alpha}\right)^{2}}{n} \frac{1}{2 f\left(\xi_{\alpha}\right)}+\frac{\left(N_{\beta}-k_{\beta}\right)^{2}}{n} \frac{1}{2 f\left(\xi_{\beta}\right)}+\rho_{n}
\end{aligned}
$$

where

$$
\rho_{n}=\left(\frac{1}{n}\left|N_{\alpha}-k_{\alpha}\right|^{3 / 2}+\frac{1}{n}\left|N_{\beta}-k_{\beta}\right|^{3 / 2}\right) r_{n 1}+\left(\frac{1}{n^{2}}\left(N_{\alpha}-k_{\alpha}\right)^{2}+\frac{1}{n^{2}}\left(N_{\beta}-k_{\beta}\right)^{2}\right) r_{n 1}^{2} .
$$

By Bernstein's inequality $P\left(\left|\pi_{1}\right|>c \sqrt{n \ln n}\right)=O\left(n^{-d}\right)$, as $n \rightarrow \infty$. If in $(24) \ell \geqslant 2$ then we can clearly bound $\left|\pi_{\ell}\right|$ by $c n$ for some positive constant $c$ not depending on $n$. This argument shows that for any $r \geqslant 3$ and all non-negative integers $i_{1}, \ldots, i_{r}$ satisfying $\sum_{\ell=1}^{r} \ell i_{\ell}=r$

$$
P\left(\sqrt{n}\left|\prod_{\ell=1}^{r}\left(n^{-\ell} \pi_{\ell}\right)^{i_{\ell}}\right|>c(\ln n)^{3 / 2} n^{-1}\right)=O\left(n^{-d}\right)
$$

as $n \rightarrow \infty$. This proves (27).

Further consider $\pi_{1}^{2}-\pi_{2}$ in (26). From (24) and (28) we have

$$
\begin{aligned}
\frac{1}{n \sqrt{n}}\left(\pi_{1}^{2}-\pi_{2}\right)=\frac{2}{n \sqrt{n}} & \sum_{1 \leqslant i<j \leqslant n}\left(W_{i}-v\right)\left(W_{j}-v\right) \\
& +\frac{1}{\sqrt{n}}\left[\alpha\left(\xi_{\alpha}-\mu\right)^{2}+(1-\beta)\left(\xi_{\beta}-\mu\right)^{2}-(v-\mu)^{2}\right]+\bar{\rho}_{n}
\end{aligned}
$$


where $P\left(\left|\bar{\rho}_{n}\right|>c(\ln n)^{3 / 2} n^{-1}\right)=O\left(n^{-d}\right)$.

Finally, we obtain the representation for $\pi_{1}$ from (28):

$$
\begin{aligned}
\frac{1}{\sqrt{n}} \pi_{1}=\frac{1}{\sqrt{n}} \sum_{i=1}^{n}( & \left.W_{i}-v\right)-\frac{1}{n \sqrt{n}} \sum_{1 \leqslant i<j \leqslant n}\left(I\left(X_{i} \leqslant \xi_{\alpha}\right)-\alpha\right)\left(I\left(X_{j} \leqslant \xi_{\alpha}\right)-\alpha\right) \frac{1}{f\left(\xi_{\alpha}\right)} \\
& +\frac{1}{n \sqrt{n}} \sum_{1 \leqslant i<j \leqslant n}\left(I\left(X_{i} \leqslant \xi_{\beta}\right)-\beta\right)\left(I\left(X_{j} \leqslant \xi_{\beta}\right)-\beta\right) \frac{1}{f\left(\xi_{\beta}\right)} \\
& +\frac{1}{n \sqrt{n}}\left(\left|\sum_{i=1}^{n}\left(I\left(X_{i} \leqslant \xi_{\alpha}\right)-\alpha\right)\right|^{3 / 2}+\left|\sum_{i=1}^{n}\left(I\left(X_{i} \leqslant \xi_{\beta}\right)-\beta\right)\right|^{3 / 2}\right) r_{n 1} \\
& +\frac{1}{\sqrt{n}}\left[\left(\xi_{\alpha}-\mu\right) w_{\alpha}-\left(\xi_{\beta}-\mu\right) w_{\beta}-\frac{\alpha(1-\alpha)}{2 f\left(\xi_{\alpha}\right)}+\frac{\beta(1-\beta)}{2 f\left(\xi_{\beta}\right)}\right]+\bar{r}_{n 1},
\end{aligned}
$$

where $P\left(\left|\widetilde{r}_{n 1}\right|>c(\ln n)^{3 / 2} n^{-1}\right)=O\left(n^{-d}\right)$.

Combining (26)-(27) and (29)-(30) we obtain the proof of Lemma 2.

ProOF OF THEOREM 1: Using the notation of Lemma 2, let $\varphi(t)=E \exp \left(i t g\left(X_{1}\right)\right)$, $t \in R$,

$$
\begin{aligned}
\bar{F}_{\sigma}(x) & =P\left\{U_{1}+U_{2} \leqslant x\right\} \quad \text { and }, \\
\bar{G}(x) & =\Phi(x)-\frac{1}{\sigma^{3} \sqrt{n}} \frac{\kappa_{3}}{6} \phi(x)\left(x^{2}-1\right), x \in R,
\end{aligned}
$$

where $\kappa_{3}=E g^{3}\left(X_{1}\right)+3 E g\left(X_{1}\right) g\left(X_{2}\right) h\left(X_{1}, X_{2}\right)$. Simple calculations show that

$$
|\varphi(t)| \leqslant 1-(\beta-\alpha)+2|t|^{-1}, t \in R
$$

and if $|t|>2 /(\beta-\alpha)$ then $|\varphi(t)|<1$ and hence the Cramér condition is satisfied. Since the functions $g$ and $h$ are bounded then the theorem giving the asymptotic expansion for $U$-statistics holds (see, for example, $[\mathbf{2}, \mathbf{5}, \mathbf{1 8}]$ )

$$
\sup _{x}\left|\bar{F}_{\sigma}(x)-\bar{G}(x)\right|=O\left(n^{-1}\right) .
$$

Now we shall apply Lemma 2. First we note that

$$
P\left(\left|\sum_{i=1}^{n}\left(I\left(X_{i} \leqslant \xi_{\gamma}\right)-\gamma\right)\right|>c \sqrt{n \ln n}\right)=O\left(n^{-d}\right)
$$

for $\gamma=\alpha$ and $\beta$. Therefore

$$
F_{\sigma}(x)=\bar{F}_{\sigma}\left(x-\frac{\lambda_{3}}{6 \sqrt{n}}+O\left((\ln n)^{5 / 4} n^{-3 / 4}\right)\right)+O\left(n^{-1}\right) .
$$

And from

$$
\begin{aligned}
\sup _{x}\left|F_{\sigma}(x)-G(x)\right| \leqslant \sup _{x}\left|\bar{F}_{\sigma}(x)-\bar{G}(x)\right|+O\left(n^{-1}\right) & \\
& \quad+\sup _{x}\left|G(x)-\bar{G}\left(x-\frac{\lambda_{3}}{6 \sqrt{n}}+O\left((\ln n)^{5 / 4} n^{-3 / 4}\right)\right)\right| \\
= & O\left((\ln n)^{5 / 4} n^{-3 / 4}\right)
\end{aligned}
$$


we obtain the proof of Theorem 1.

Proof of Theorem 2: By Bahadur's theorem (see, for example, [1], or [11])

$$
X_{n k_{\gamma}}=\xi_{\gamma}-\frac{N_{\gamma}-\gamma n}{n} \frac{1}{f\left(\xi_{\gamma}\right)}+\rho_{n},
$$

where

$$
P\left(\left|\rho_{n}\right|>c(\ln n / n)^{3 / 4}\right)=O\left(n^{-d}\right)
$$

for some $c>0$ and every $d>0$ not depending on $n$. Furthermore, with the help of (11), (13), (21) and (23) for $p=2$ and (34) with $\gamma=\alpha, \beta$ we obtain the following representation for $S_{n}^{2}$

$$
S_{n}^{2}=\sigma^{2}+\frac{1}{n} \sum_{i=1}^{n} \psi\left(X_{i}\right)+\tilde{\rho}_{n}
$$

where

$\psi\left(X_{i}\right)=\left(I\left(X_{i} \leqslant \xi_{\alpha}\right)-\alpha\right) \frac{2 \alpha}{f\left(\xi_{\alpha}\right)}\left(v-\xi_{\alpha}\right)+\left(\left(W_{i}-v\right)^{2}-\sigma^{2}\right)+\left(I\left(X_{i} \leqslant \xi_{\beta}\right)-\beta\right) \frac{2 \beta}{f\left(\xi_{\beta}\right)}\left(v-\xi_{\beta}\right)$, and the remainder term $\widetilde{\rho}_{n}$ satisfies (35). The details of the proof are similar to the proofs in [13] and [11]. Here we omit the details since the proof follows that of Lemma 2.

Recall

$$
P\left(\left|\sum_{i=1}^{n}\left(I\left(X_{i} \leqslant \xi_{\gamma}\right)-\gamma\right)\right|>c \sqrt{n \ln n}\right)=O\left(n^{-d}\right)
$$

for $\gamma=\alpha$ and $\beta$. Applying this observation to the representation in Lemma 2 and using (36) we can write (7) as

$$
F_{s}(x)=P\left(\frac{U_{1}+U_{2}+\lambda_{3} /(6 \sqrt{n})+O\left((\ln n)^{5 / 4} n^{-3 / 4}\right)}{\sqrt{1+\left(\sigma^{2} n\right)^{-1} \sum_{i=1}^{n} \psi\left(X_{i}\right)+\sigma^{-2} \widetilde{\rho}_{n}}} \leqslant x\right)+O\left(n^{-1}\right)
$$

as $n \rightarrow \infty$. By the inequality from $[16]$

$$
\left(1+\left(\sigma^{2} n\right)^{-1} \sum_{i=1}^{n} \psi\left(X_{i}\right)+\sigma^{-2} \tilde{\rho}_{n}\right)^{-1 / 2}=1-\frac{1}{2 \sigma^{2} n} \sum_{i=1}^{n} \psi\left(X_{i}\right)+O\left((\ln n / n)^{3 / 4}\right)
$$

with probability $1-O\left(n^{-d}\right)$ for every $d>0$. Thus we can write

$$
\begin{array}{r}
F_{s}(x)=P\left(\left(U_{1}+U_{2}\right)\left(1-\frac{1}{2 \sigma^{2} n} \sum_{i=1}^{n} \psi\left(X_{i}\right)\right)+\frac{\lambda_{3}}{6 \sqrt{n}}+O\left((\ln n)^{5 / 4} n^{-3 / 4}\right) \leqslant x\right) \\
+O\left(n^{-1}\right), \quad x \in R .
\end{array}
$$

Here with probability $1-O\left(n^{-d}\right)$ for every $d>0$ 


$$
\left(U_{1}+U_{2}\right)\left(1-\frac{1}{2 \sigma^{2} n} \sum_{i=1}^{n} \psi\left(X_{i}\right)\right)=U_{1}+U_{3}-\frac{1}{2 \sigma^{3} \sqrt{n}} E g\left(X_{1}\right) \psi\left(X_{1}\right)+O\left((\ln n)^{3} n^{-1}\right)
$$

where

$$
U_{3}=\frac{1}{\sigma n \sqrt{n}} \sum_{1 \leqslant i<j \leqslant n} \bar{h}\left(X_{i}, X_{j}\right)
$$

and

Therefore

$$
\bar{h}\left(X_{i}, X_{j}\right)=h\left(X_{i}, X_{j}\right)-\frac{1}{2 \sigma^{2}}\left(g\left(X_{i}\right) \psi\left(X_{j}\right)+g\left(X_{j}\right) \psi\left(X_{i}\right)\right) .
$$

$$
\begin{gathered}
F_{s}(x)=P\left(U_{1}+U_{3}+\lambda \leqslant x\right)+O\left(n^{-1}\right) \\
\text { with } \lambda=-\frac{1}{2 \sigma^{3} \sqrt{n}} E g\left(X_{1}\right) \psi\left(X_{1}\right)+\frac{\lambda_{3}}{6 \sqrt{n}}+O\left((\ln n)^{5 / 4} n^{-3 / 4}\right) . \text { Denote } \\
\bar{H}(x)=\Phi(x)-\frac{1}{\sqrt{n}} \frac{\bar{\kappa}_{3}}{6} \phi(x)\left(x^{2}-1\right), \quad x \in R \\
\bar{\kappa}_{3}=\left[E g^{3}\left(X_{1}\right)+3 E g\left(X_{1}\right) g\left(X_{2}\right) \bar{h}\left(X_{1}, X_{2}\right)\right] \sigma^{-3} .
\end{gathered}
$$

Further by analogy with (31) - (33) we have after simple calculations

$$
\begin{aligned}
\sup _{x}\left|F_{s}(x)-H(x)\right| & =\sup _{x}\left|P\left(U_{1}+U_{3} \leqslant x\right)-H(x+\lambda)\right|+O\left(n^{-1}\right) \\
& \leqslant \sup _{x}\left|P\left(U_{1}+U_{3} \leqslant x\right)-\bar{H}(x)\right|+\sup _{x}|\bar{H}(x)-H(x+\lambda)|+O\left(n^{-1}\right) \\
& =O\left((\ln n)^{5 / 4} n^{-3 / 4}\right) .
\end{aligned}
$$

This proves Theorem 2 .

\section{REFERENCES}

[1] R.R. Bahadur, 'A note on quantiles in large samples', Ann. Math. Statist. 37 (1966), $577-580$.

[2] V. Bentkus, F. Götze, and W.R. van Zwet, 'An Edgeworth expansion for symmetric statistics', Ann. Statist. 25 (1997), 851-896.

[3] P.J. Bickel, 'On some robust estimates of location', Ann. Math. Statist. 36 (1965), 847-858.

[4] Y.V. Borovskikh, U-statistics in Banach spaces (VSP, Utrecht, The Netherlands, 1996).

[5] Y.V. Borovskikh, Sharp estimates of the rate of convergence for $U$-statistics, (Report 98-21) (School of Mathematics and Statistics, University of Sydney, 1998).

[6] Y.V. Borovskikh and N.C. Weber, 'Large deviation results for a $U$-statistical sum with product kernel', Bull. Austral. Math. Soc. 63 (2001), 151-165.

[7] L.E. Dickson, Elementary theory of equations (Wiley, New York, 1914).

[8] A.J. van Es, 'On the weak limits of elementary symmetric polynomials', Ann. Probab. 14 (1986), 677-695. 
[9] A.J. van Es and R. Helmers, 'Elementary symmetric polynomials of increasing order', Probab. Theory Related Fields 80 (1986), 21-35.

[10] A.J. van Es, R. Helmers and M. Huskova, 'On a crossroad of resampling plans: bootstrapping elementary symmetric polynomials', Statist. Neerlandica 54 (2000), 100-110.

[11] N. Gribkova and R. Helmers, 'The empirical Edgeworth expansion for a studentized trimmed mean', (CWI Report PNA-RO214, (http://db.cwi.nl/rapporten/), Probability Networks and Algorithms (2002), 1-21.

[12] G. Halasz and G.J. Szekely, 'On the elementary symmetric polynomials of independent random variables', Acta Math. Acad. Sci. Hung. 28 (1976), 397-400.

[13] P. Hall and A.R. Padmanabhan, 'On the bootstrap and the trimmed mean', J. Multivariate Anal. 41 (1992), 132-153.

[14] R. Helmers, 'Edgeworth expansions for trimmed linear combinations of order statistics', in Proceedings of the Second Prague Symp. Asymptotic Statist., (P. Mandl and M. Huskova, Editors) (North-Holland, Amsterdam, 1979), pp. 221-232.

[15] R. Helmers, 'A Berry-Esseen theorem for linear combinations of order statistics', Ann. Probab. 9 (1981), 342-347.

[16] W. Hoeffding, 'Probability inequalities for sums of bounded random variables', J. Amer. Statist. Assoc. 58 (1963), 13-30.

[17] P. Janssen, R. Serfling and N. Veraverbeke, 'Asymptotic normality of $U$-statistics based on trimmed samples', J. Statist. Plann. Inference 16 (1987), 63-74.

[18] B.Y. Jing and Q. Wang, 'Edgeworth expansion for $U$-statistics under minimal conditions', Ann. Statist. 31 (2003), 1376-1391.

[19] H. Putter and W.R. van Zwet, 'Empirical Edgeworth expansions for symmetric statistics', Ann. Statist. 26 (1998), 1540-1569.

[20] R.D. Reiss, Approximate distributions of order statistics (Springer-Verlag, New York, 1989).

[21] G.J. Szekely, 'A limit theorem for elementary symmetric polynomials of independent random variables', Z. Wahrsch. Verw. Gebiete 59 (1982), 355-359.

[22] T. de Wet, 'Berry-Esseen results for the trimmed mean', South African Statist. J. 10 (1976), 77-96.

[23] T. de Wet and J.H. Venter, 'An asymptotic representation of trimmed means with applications', South African Statist. J. 8 (1974), 127-133.

[24] W.R. van Zwet, 'Asymptotic expansions for the distribution functions of linear combinations of order statistics', in Statistical decision theory and related topics II, (D.S. Moore and S.S. Gupta, Editors) (Academic Press, New York, 1977), pp. 421-437.

[25] W.R. van Zwet, 'The Edgeworth expansion for linear combinations of uniform order statistics', in Proceedings of the Second Prague Symp. Asymptotic Statist., (P. Mandle and M. Huskova, Editors) (North-Holland, Amsterdam, 1979), pp. 93-101.

Department of Applied Mathematics

Transport University

Moskovsky Avenue, 9

190031 St Petersburg

Russia
School of Mathematics and Statistics F07

University of Sydney

New South Wales 2006

Australia 